\title{
The Anti-Bacterial Effect of Punica granatum Extracts Against Antibiotic Resistant Pasteurella haemolytica
}

\author{
Shaza Anwar Al Laham ${ }^{1, *}$, Frdoos Mohammad Al Fadel ${ }^{1}$ \\ ${ }^{1}$ Pharmacology and Toxicology Department, Faculty of Pharmacy, Damascus University, Damascus, Syria \\ *Corresponding author: Shaza Anwar Al Laham, Pharmacology and Toxicology Department, Faculty of Pharmacy, Damascus University, Damascus, Syria. Tel: +96-30113136591, Fax: \\ +96-30113312041, E-mail: Lahamshaza@gmail.com, sh-laham@scs-net.org.
}

Received: August 18, 2012; Revised: October 28, 2012; Accepted: December 8, 2012

\begin{abstract}
Background: Continuous researches on drugs that solve the problem of growing number of resistant bacteria are important.
Objectives: This research aims to investigate the effectiveness of anti-bacterial properties shown by the extracts prepared from different parts of Punica granatum L. (punicaceae), grown in Syria, against Pasteurella haemolytica, which are resistant to all studied antibiotics. Materials and Methods: A total number of 504 samples of dead sheep lungs were investigated for detection of P. haemolytica, using blood agar, blue methylene, and biochemical tests (oxidase, catalase, indole, urease). Different parts of P. granatum (pericarp, leaves, flowers, seeds) were extracted by water, absolute alcohol, and ether using soxhlet device and rotary vacuum evaporator. Antibiotic susceptibility testing for $P$. haemolytica by Kirby-Bauer disk diffusion method was conducted, then the extracts susceptibility test against $P$. haemolytica was conducted.

Results: $P$. haemolytica has infected $20.63 \%$ of the total number of samples. The alcoholic extracts prepared from different parts of $P$. granatum showed the high antibacterial effectiveness, the pericarp extract was the best, whereas the water and ether petroleum extracts had no antibacterial effectiveness against resistant $P$. haemolytica.

Conclusions: Ethanol extracts of P. granatum (pericarp, leaves, flowers, seeds) have antibacterial effects against $P$. haemolytica which has shown resistance to all studied antibiotics.
\end{abstract}

Keywords: Punica granatum; Pasteurella haemolytica; Resistant Bacteria; Antibiotics

\section{Background}

Punica granatum Linn (pomegranate) belonging to family of Punicaceae. The medicinal parts are the root, bark, fruits, peel of the fruit and the flowers (1). With regards to the popular therapeutic uses of pomegranate, it has known as an anti-diarrhea, antiparasitic agent for treatment of ulcers, diuretic, with antibacterial activity (2). It is widely used as an antipyretic analgesic in Chinese culture (3). Various parts of the Pomegranate, havebeen used for various medicinal purposes $(1,4)$. The fruit is good for dysentery, diarrhea and gastralgia. The extract from different parts of the fruit showed antibacterial activity (3). Although many studies have reported the antibacterial activity of pomegranate (5-7), but not enough studies have reported its effect on bacterial resistance, and had not determined the most effective part of the plant in dealing with bacteria, whether peel of the fruit, leaves, flowers, or seeds of the pomegranate.

Pasteurella multocida and Mannheimia (Pasteurella) haemolytica are causative agents of several economically significant veterinary diseases occurring in numerous species such as: cattle, buffaloes, sheep, goats, poultry, turkeys, rabbits, horses and camels. M. haemolytica is the causative agent of shipping fever or pneumonic pasteurellosis (8) and respiratory diseases in cattle, including bronchopneumonia in feedlot cattle. The species usually invovled in these infections are P. multocida sp. multocida sp. septica, P. canis, P. dagmatis, and P. stomatis. Most human infections are associated with Pasteurella sp infrction resulted from animal bites (9).

\section{Objectives}

Unfortunately, the resistance to antibiotics is increasing, and these bacteria showed resistance to many antibiotics (10-12). So in our investigation, we tried to discover the possibility of owning the plant capable to respond to these bacteria, and we believe that, this is the first study describing the antibacterial activity of $P$. granatum extracts against $P$. haemolytica, and we hope that its results to be a starting point in administering the plant extracts

Implication for health policy/practice/research/medical education:

Unfortunately, the resistance to antibiotics is increasing, so we tried in our investigation to discover the possibility of owning the plant the capacity to respond to Pasteurella haemolytica, a causative agent of several economically significant veterinary diseases, which were resistant to all studied antibiotics. We believe that, this is the first study describing the antibacterial activity by different parts of Punica granatum extracts against P. haemolytica, and we hope that its results to be a starting point in administering the plant extracts on infected animals.

Copyright @ 2013,Ahvaz JundishapurUniversity of Medical Sciences; Published by Kowsar Corp. This is an open-access article distributed under the terms of the Creative Commons Attribution License, which permits unrestricted use, distribution, and reproduction in any medium, provided the original work is properly cited. 
for infected animals.

\section{Materials and Methods}

\subsection{Collection of Plant Specimens}

Ripe fruits of pomegranate were collected from July to September 2010 from Damascus rural area (Duma), while the leaves and the flowers were collected in the early morning times from May to July 2010 from the mentioned location, which were identified by Damascus University. The peel were separated from the fruits, washed with cold distilled water, then dried with hot air at a temperature not exceeding $60^{\circ} \mathrm{C}$ in shadow. Then were crushed properly by metal mortar in order to obtain fine homogeneous powder, kept in paper bags with free humidity conditions, ready to prepare extract (13).

\subsection{Preparing Plant Extracts}

Plant parts were extracted separately by continuous extraction device (Soxhlet apparatus ), based on the method described by Wang (14), for preparing plant extracts by organic solvents, $50 \mathrm{~g}$ of plant powder were placed by an electric mortar, inside the thimble-holder of Soxhlet apparatus, with $500 \mathrm{~mL}$ of each organic solvent (rate: 10.1 weight:volume). Three different polar solvents have been selected to extract the components of the plants, which are respectively: water, absolute ethanol, petroleum ether. Extraction period took 4 hours, until the used solvent comes out of thimble colorless. Then to concentrate the extracts the ethanol, and petroleum ether extracts were dried using rotary vacuum evaporator at a temperature not exceeding $40^{\circ} \mathrm{C}$, while the aqueous extract was dried using freeze dryer. The thick layer of the bottom was stored in sterile bottles at $4^{\circ} \mathrm{C}$ for further experiments. All extracts were filter-sterilized using a $0.45 \mu \mathrm{m}$ membrane filters (Whatman Co., UK) (13).

\subsection{Sampling Method}

Samples of dead sheep were daily conducted to the morgue of the Central Laboratory of Veterinary in Damascus during 2010-2011. A total number of 504 lung biopsies samples were investigated. The samples were kept at 2 $4^{\circ} \mathrm{C}$ and transported to the laboratory in sterile tubes, fitted with a strap closure, and card number includes name of the sample, place, and date of collection.

\subsection{Cultured and Identification Methods of Patho- logical Sample}

The following information was registered on the bottom of the petri plates: the number, name of the sample, place, and date of collection. Then the platinum rod after sterilization by flame lamp was planted within the sample (biopsy lung), and passed on blood agar (HiMedia, India), and incubated for $20-24$ hours at $35-37^{\circ} \mathrm{C}$ at an aerobic culture incubator. It is worth mentioning that all samples were planted in two hours from the time of sampling. The bacteria were identified culturally, morphologically and biochemically.

Microscopic examination was conducted after 24 hours of incubation on blood agar plates using the Gram stain, immersion oil, methylene blue dye, light microscope. All of the following biochemical tests were conducted: oxidase, catalase, and indole. In addition, fermentation reactions of the following sugars: D-glucose, D-mannitol, lactose were conducted.

\subsection{Bacterial Growth Inhibition Test by the Use of Disk Diffusion Method}

The antimicrobial susceptibility testing was carried out on blood agar by disc diffusion method (Kirby-Bauer disk diffusion susceptibility test protocol) using the following antimicrobial substances (Becton Dikinson, microbiology systems, MD, USA) as described by national committee for clinical laboratory standards (15).

The $5 \mathrm{~mm}$ diameter standard discs containing certain concentrations of many antibiotics (Bioanalyse), as follows: Amikacin $(30 \mu \mathrm{g})$, Ampicillin $(10 \mu \mathrm{g})$, Cephalexin (30 $\mu \mathrm{g})$, Cephalothin $(30 \mu \mathrm{g})$, Doxycyclin $(30 \mu \mathrm{g})$, Cefadroxil $(30 \mu \mathrm{g})$, Ciprofloxacin $(5 \mu \mathrm{g})$, Clindamycin $(2 \mu \mathrm{g})$, Chloramphenicol $(30 \mu \mathrm{g})$, Erythromycin $(15 \mu \mathrm{g})$, Gentamicin $(10 \mu \mathrm{g})$, Norfloxacin $(10 \mu \mathrm{g})$, Oxytetracycline $(30 \mu \mathrm{g})$, Pefloxacin $(5 \mu \mathrm{g})$, Oxacillin $(1 \mu \mathrm{g})$, Enrofloxacin $(5 \mu \mathrm{g})$, Tetracycline $(30 \mu \mathrm{g})$ and Amoxicillin $(25 \mu \mathrm{g})$. the resistance breakpoints were defined by the national committee for Clinical Laboratory Standards (NCCLS, 2000) for Gramnegative bacteria (15).

4 - 5 colonies of bacteria were suspended (after pure isolation and identification) in the test tube containing $2 \mathrm{~mL}$ of physiological solution, mixed thoroughly until a turbid homogeneous was obtained. Sterile swab sticks immersed in suspension, and spread onto the surface of the Muller Hinton agar plates, and then the agar plates were partly covered with lids to dry before proceeding next step. The antibiotic discs were placed and gently pressed, by sterilized forceps, onto the middle of plates (Forceps was sterilized after each antibiotic), finally the agar plates were covered and incubated in aerobic incubator at $37^{\circ} \mathrm{C}$ for 24 hours. The plates were observed for the presence of inhibition of bacterial growth that was indicated by an inhibition zone around the wells. The size of the inhibition zone was measured and the antibacterial activity expressed in millimeters according to the average diameter of the inhibition zone.

\subsection{A Bacterial Growth Inhibition Test of Plant Ex- tracts Using the Disk Diffusion Method}

Sterile filter paper discs $(5 \mathrm{~mm})$ were soaked with $5 \mathrm{~mL}$ of the diluted extracts $(66 \mathrm{mg} / \mathrm{mL})$ of pericarp, leaves, flowers, seeds in ethanol, water, and petroleum ether, 
so each disc was impregnated with $0.33 \mathrm{mg} /$ tablet. All extracts were filter-sterilized using a $0.45 \mu \mathrm{m}$ membrane filters (Whatman, Co., UK). Control disks also prepared with absolute ethanol, Water, and petroleum ether. The Disks were placed in Petri dishes containing Mueller Hinton agar and incubated for 16 hours at $37^{\circ} \mathrm{C}$, after incubation, all growth inhibition zones were observed, and the diameters were measured in millimeters with a ruler. Results were expressed as the percentage of inhibition of bacterial growth, determined by comparing it with control disks, and standard susceptibility disks (14). After completing the work the petri dishes were eliminated through the autoclave.

\section{Results}

\subsection{Identification of the Bacteria}

Selected Bacteria samples gave us the following results: Gram staining: Gram-negative, non-motile, rod-shaped bacteria, short chains organizing, compatible with reference (9). Methylene blue staining: bipolar staining, and mucous capsular membranes observed which were compatible with reference (9).

\subsection{Colony and Cultural Characters}

On standard agar plates of fastidious Gram-negative bacteria like blood agar, regular, smooth, convex, gray, nontransparent, circular, and small colonies with 0.5 $2 \mathrm{~mm}$ diameter were appeared. These results complied with (9).

\subsection{The Results of Biochemical Tests}

The results were shown in Table 1, These results conformed with Selmi et al. findings ( 9 ). So in 104 (20.63\%) of 504 samples, P. haemolytica were detected.

\subsection{Antimicrobial Susceptibility Results Against Pasteurella haemolytica}

Bacterial colonies were resistant to the studied antibiotics, since all the inhibition zone diameters were lower than the estimated values for each antibiotics, based on the NCCLS2000 criteria (15), and to the standard leaflet of antibiotic discs from the manufacturer.

\begin{tabular}{ll}
\hline Table 1. The Biochemical Tests Results for P. haemolytica \\
\hline Test & Result \\
\hline Oxidase & positive \\
Catalase & positive \\
Indol & negative \\
D-mannitol & positive \\
D-glucose & positive \\
Lactose & positive \\
Urease & negative \\
\hline
\end{tabular}

\subsection{Antibacterial Effect of Plant Extracts}

As shown in Table 2, the extracts from different parts of the plant (pericarp, leaves, flowers, seeds) had antibacterial activity against $P$. haemolytica, with the inhibition zone ranging from 10 to $25 \mathrm{~mm}$. The results indicated the presence of a $24 \mathrm{~mm}$ inhibition zone of ethanol extract of pericarp (the distinctive zones of inhibition), while the ethanol extract of leaves were $14 \mathrm{~mm}, 12.3 \mathrm{~mm}$ for flowers, and $9.8 \mathrm{~mm}$ for seeds extracts. The most active extracts were those obtained from the flowers of $P$. granatum (98.5\%). The organic solvent ether, and water extract from all parts of the plants were not active against P. haemolytica (without any inhibition zone).

Table 2. Antibacterial Activity of Different Extracts of Studied Plants Against P. haemolytica

\begin{tabular}{|c|c|c|c|c|}
\hline \multirow[t]{2}{*}{ P. granatum } & \multicolumn{3}{|c|}{ Inhibition Zone of Plant Extracts mm, Mean \pm SD } & \multirow[t]{2}{*}{ Sensitive Bacteria, \% } \\
\hline & Water Extract & Ether Petroleum Extract & Ethanol Extract & \\
\hline Control $/ 5 \mu \mathrm{m}$ & 0 & 0 & 0 & 0 \\
\hline Pericarp & 0 & 0 & $24 \pm 1.2$ & 97.6 \\
\hline Leaves & 0 & 0 & $14 \pm 0.9$ & 96.4 \\
\hline Flowers & 0 & 0 & $12.3 \pm 1.1$ & 98.5 \\
\hline Seeds & 0 & 0 & $9.8 \pm 0.87$ & 95.3 \\
\hline
\end{tabular}

\section{Discussion}

M. haemolytica serotype 1 is the primary causative agent responsible for bovine pneumonic mannheimiosis, also known as shipping fever in cattle. The bacterium produces a variety of virulence factors, foremost of which is the exotoxic leukotoxin (16). The outer membrane proteins and capsular material including hyaluronic acid of serotype A have been characterized and are probably related with virulence by interacting with host immune system (9). The present study showed that the mortality rate in sheeps with P. haemolytica infection was $20.63 \%$, nearly $12 \%$ in the results of Cecile study (12).

P.haemolytica was resistant to 18 tested antibiotics. The increasing resistance in other studies against this type of bacteria of traditional antibiotics such as Chloramphenicol (12), Penicillin, Cephalotin, Enrofloxacin (17), Tetracyclin (18), Oxytetracycline, Doxycycline (19), Erythromycin, 
and Penicillin (20), has confirmed our findings. However, several studies demonstrated the Ciprofloxacin effectiveness against this bacteria (21), Amikacin, and Gentamicin (20), of which we have not examined in our study.

That directs us that there is an urgent need to develop alternative antimicrobial drugs against this type of bacteria, so we used $P$. granatum with different extracts. The results showed that $P$. granatum possesses strong antibacterial activities against Gram-negative P. haemolytica, and this is reported for the first time. Previous studies have also demonstrated that $P$. granatum L. has the ability to inhibit the activity of Staphylococcus aureus (Gram positive), Escherichia coli, Klebsiella pneumoniae, Proteus vulgaris, and Salmonella typhi (Gram negative) organisms (3, 4). Although this study (4) showed that the methanol and water extracts of the P. granatum L. leaves, peel have antibacterial activity, but our study showed an opposite result for water extract. Both aqueous and ether petroleum extracts of different parts of the plant (pericarp, leaves, flowers, seeds) did not have antibacterial effect, while ethanol extracts produced disparate inhibition zone for P. haemolytica, that was ranging from 10 to $25 \mathrm{~mm}$.

Although the previous studies pointed out the impacts of flowers, in decreasing the blood glucose (22), and reducing the cholesterol or triglyceride serum level (23), which have analgesic (24), and anti-allergic effect (25), but it did not show the antibacterial effects, while our study proved it in the alcoholic extract which had caused $12.3 \mathrm{~mm}$ inhibition zone. In the present study, the ethanol extracts of the seeds had antibacterial activity, which was the lowest effect comparative to other studied parts. This result supports the findings of Al-Zoreky study (26), and Abdollahzadeh (27). While the difference was in the water extract which had antimicrobial effects $(28,29)$.

It was found that $P$. granatum peels contain tannins, anthocynins, flavonoids, pectins, three estrogen compounds luteolin, quercetin and kaempferol (4). P. granatum contains large amount of tannins (25\%) and the antibacterial activity may be indicating the presence of some secondary metabolites. The antibacterial activity may indicate the presence of some metabolic toxins or broad-spectrum antibiotic compounds (3). The tannins makes it useful as an astringent for sore throats, diarrhea and dysentery, but the drugs containing tannins and alkaloids, are anthelmintic and amoeboid (30). Pomegranate fruit peel compounds tannins, piperidine alkaloids and pelletierin triggers like strychnine, a raised stimulant reflex, which can escalate to tetanus and is effective against diverse tapeworms, ring worms and nematodes infections (30). Peels of P. granatum L. also include a wide variety of phytochemical compounds, e.g., gallotannins, ellagic acid, gallagic acid, punicalins, punicalagins, and this fruit is found to be a rich source of polyphenolic compounds. The antibacterial effect may be due to phenolic compounds which could increase the concentration of organic acids (31).

The ethanol extracts of the P. granatum revealed differ- ent antibacterial activity against $P$. haemolytica, which affected sheep, and had shown completely antibiotics resistance. While the water and ether extracts had no antibacterial effectiveness, the antibacterial effect may be caused by many compounds.

\section{Acknowledgements}

The authors sincerely thanked the Central Laboratory of Veterinary in Damascus for their kind assistant, and hard efforts and a special thanks to Prof. Dr. Anwar Al-Khatib from Damascus University.

\section{Authors' Contribution}

$50 \%$ for each one .

\section{Financial Disclosure}

There is no Financial Disclosure.

\section{Funding/Support}

There is no Funding/Support.

\section{References}

1. Evans William Charles. Trease and Evans' pharmacognosy.: Elsevier Health Sciences; 2009.

2. Pradeep BV, Manojbabu MK, Palaniswamy M. Antibacterial Activity of Punica granatum L. Against Gastro Intestinal Tract Infection Causing Organisms. Ethnobotanic Leafl. 2008;2008(1):143.

3. Lee Chia-Jung, Chen Lih-Geeng, Liang Wen-Li, Wang Ching-Chiung. Anti-inflammatory effects of Punica granatum Linne in vitro and in vivo. Food Chemistry. 2010;118(2):315-322.

4. Rathinamoorthy R, Thilagavathi G. Antimicrobial and In-Vitro Drug Release Studies of Microencapsulated Terminalia chebula extract finished Fabric. Int JPharm Life Sci. 2011;2(10):1147-1153.

5. Choi Jang-Gi, Kang Ok-Hwa, Lee Young-Seob, Chae Hee-Sung, Oh You-Chang, Brice Obiang-Obounou, et al. In Vitro and In Vivo Antibacterial Activity of Punica granatum Peel Ethanol Extract against Salmonella. Evidence-Based Complement Alt Med. 2011;2011.

6. Duman AD, Ozgen M, Dayisoylu KS, Erbil N, Durgac C. Antimicrobial activity of six pomegranate (Punica granatum L.) varieties and their relation to some of their pomological and phytonutrient characteristics. Molecules. 2009;14(5):1808-17.

7. Nair R, Chanda S. Antibacterial activity of Punica granatum in different solvents. Indian J pharm sci. 2005;67(2):239-243.

8. Sleim R. Review: Major pathogenic components of Pasteurella multocida and Mannheimia (Pasteurella) haemolytica isolated from animal origin. El Cairo, Egypt: Bacteriology Department, Animal Health Research Institute. 2005.

9. Garrity GM, Brenner DJ, Krieg NRStaley, JT. Bergey's Manual of Systematic Bacteriology. 2 ed: Library of Congress Cataloging-InPublication Data; 2005.

10. Chang WH, Carter GR. Multiple drug resistance in Pasteurella multocida and Pasteurella haemolytica from cattle and swine. $J$ Am Vet Med Assoc. 1976;169(7):710-2.

11. Malik YS, Chander Y, Gupta SC, Goyal SM. A Retrospective Study on Antimicrobial Resistance in Mannheimia (Pasteurella) haemolytica, Escherichia coli, Salmonella Species, and Bordetella avium from Chickens in Minnesota. J Appl Poultry Res. 2005;14(3):506-511.

12. Vassort-Bruneau Cécile, Lesage-Descauses Marie-Claude, Martel Jean-Louis, Lafont Jean-Pierre, Chaslus-Dancla Elisabeth. CAT III chloramphenicol resistance in Pasteurella haemolytica and Pasteurella multocida isolated from calves. J Antimicrob Chemother. 1996;38(2):205-213. 
13. Radulović Niko, Stankov-Jovanović Vesna, Stojanović Gordana, Šmelcerović Andrija, Spiteller Michael, Asakawa Yoshinori. Screening of in vitro antimicrobial and antioxidant activity of nine Hypericum species from the Balkans. Food Chem. 2007;103(1):15-21.

14. Wang Lijun, Weller Curtis L. Recent advances in extraction of nutraceuticals from plants. Trends in Food Sci Technol. 2006;17(6):300-312.

15. National Committee for Clinical Laboratory Standards . NCCLS Antimicrobial Susceptibility Testing.: National Committee for Clinical Laboratory Standards; 1992.

16. Thumbikat P, Briggs RE, Kannan MS, Maheswaran SK. Biological effects of two genetically defined leukotoxin mutants of Mannheimia haemolytica.Microbial Pathogenesis. 2003;34(5):217226.

17. Hormansdorfer S, Bauer J. [Resistance of bovine and porcine Pasteurella to florfenicol and other antibiotics]. Berl Munch Tierarztl Wochenschr. 1998;111(11-12):422-6.

18. Post KW, Cole NA, Raleigh RH. In Vitro Antimicrobial Susceptibility of Pasteurella Haemolytica and Pasteurella Multocida Recovered from Cattle with Bovine Respiratory Disease Complex.J Vet Diag Invest. 1991;3(2):124-126.

19. Mevius DJ, Breukink HJ, van Miert AS. In vitro activity of flumequine in comparison with several other antimicrobial agents against five pathogens isolated in calves in The Netherlands. Vet Q.1990;12(4):212-20.

20. Malik YS, Olsen K, Chander Y, Goyal SM. Antimicrobial Resistance in Bacterial Pathogens Isolated From Turkeys in Minnesota From 1998 to 2002. Int J Appl Res Vet Med. 2009;1(3).

21. Prescott JF, Yielding KM. In vitro susceptibility of selected veterinary bacterial pathogens to ciprofloxacin, enrofloxacin and norfloxacin. Can J Vet Res. 1990;54(1):195-7.

22. Jafri MA, Aslam M, Javed Kalim, Singh Surender. Effect of Punica granatum Linn. (flowers) on blood glucose level in normal and alloxan-induced diabetic rats. J Ethnopharm. 2000;70(3):309-314.

23. Bagri P, Ali M, Aeri V, Bhowmik M, Sultana S. Antidiabetic effect of Punica granatum flowers: effect on hyperlipidemia, pancreatic cells lipid peroxidation and antioxidant enzymes in experimental diabetes. Food Chem Toxicol. 2009;47(1):50-4.

24. Chakraborthy GunoSindhu. Analgesic activity of various extracts of Punica granatum (Linn) flowers. Int I Green Pharm. 2008;2(3):145.

25. Barwal SB, Sunil A Nirmal, Dhasade VV, Patil MJ, Pal SC, Subhash C Mandal. Antihistaminic effect of various extracts of Punica granatum Linn. flower buds. JYoung Pharm. 2009;1(4):322.

26. Al-Zoreky NS. Antimicrobial activity of pomegranate (Punica granatum L.) fruit peels. Int J Food Microbiol. 2009;134(3):244-8.

27. Abdollahzadeh Sh, Mashouf RY, Mortazavi H, Moghaddam MH, Roozbahani N, Vahedi M. Antibacterial and antifungal activities of Punica granatum peel extracts against oral pathogens. J Dent. 2011;8(1):1-6.

28. Khan AJ, Hanee Sonali. Antibacterial properties of Punica granatum peels. Int J Appl Biol Pharm Technol. 2011;2:23-27.

29. Parra Valeria I Ruiz, Gaudioso Cristina, Cecilia Marta, Silva Clara. Evaluation of the Antimicrobial activity of Aqueous Pomegranate (Punica granatum L.) extract against Shigella. Res J Biol Sci. 2011;6(5):205-212.

30. Fleming Thomas. PDR for herbal medicines.: Medical Economics Company; 1998.

31. Orak H Hülya, Demirci A, Gümüș T. Antibacterial and antifungal activity of pomegranate (Punica granatum L. cv.) peel. J Environ, Agr Food Chem. 2011;10(3):1958-1969. 